\title{
On the Growth of the Mandible
}

\author{
GEOFFREY F. WALKER AND CHARLES J. KOWALSKI \\ Department of Oral Biology, University of Michigan, \\ Ann Arbor, Michigan 48104
}

\begin{abstract}
In a cross-sectional cephalometric study of over 800 normal white American children we show that the average ANB angle is roughly twice as large as the currently employed norms for that variable. The angle is relatively the same in females from 6 to 20 years of age, but there is a definite tendency for older males to exhibit a smaller ANB angle. The dynamics of this decrease is essentially the fact that, in the male, the mandible continues to grow steadily (relative to the maxilla and nasion) throughout much of the postpuberal stage, whereas this tendency is not exhibited in the female.
\end{abstract}

The mandible and maxilla, and the relationship between these structures, have long been recognized as important components of craniofacial morphology, both in the study of racial variations and in the diagnosis and treatment of dentofacial anomalies. It is, therefore, of considerable importance to derive variables which reflect this relationship and to produce normative values for these variables in various population groups. Many studies of facial angles and proportions designed to measure this relationship have been made but, with few exceptions, the sample sizes were small with pooled ages and sexes. A case in point is the angle defined by the points subspinale (Downs' point A), nasion and supramentale (Downs' point B), commonly referred to as the ANB angle in the orthodontic literature. This angle is of particular importance in cephalometric analyses since the lines NA and $\mathrm{NB}$ are readily located in a lateral head film and provide convenient reference lines from which to measure incisal positions and inclinations. Steiner (59) chose a set of craniofacial norms, ". . . which express our concept of a normal average American child of average age," the norm for the ANB angle being set at $2^{\circ}$. He implored the reader to, "Please bear in mind that these are rough estimates, to be used as a starting point from which to vary and must be modified by other factors, not only pogonion to the line $\mathrm{NB}$, but also age, sex, race, growth potential and individual variations within these and other groupings," but provided little insight into how these modifications should be accomplished. In this paper we investigate, using cephalometric methods, the distribution of the ANB angle in a large sample of "normal" individuals for several age groups and both sexes. The intent is to test the hypothesis that an ANB angle of $2^{\circ}$ is "normal" and to investigate the dependence of this angle on age and sexual dimorphism.

\section{METHODS AND MATERIALS}

The cephalograms of children presenting "normal" dental occlusion were obtained as part of a study of normal growth conducted at the Philadelphia Center for Research in Child Growth between 1948 and 1968, and were selected from a group of 2500 white elementary and secondary school children. The project director, Dr. W. M. Krogman, “. . . took in substance children who were in "good medical health" and who had no more than the usual so-called mild 'childhood illnesses' ... "good dental health" . . . a low DMF index . . . and all four first permanent molars in place." The socioeconomic distribution of these children was concentrated in the mid-level stratum. Their ethnic distribution was Northern European (German, Scandinavian), Southern European (Italian) and Scotch, Irish and English. Middle and Eastern Europe were represented by children of Galician, Ukranian, Polish (largely Ashkenazic Jews) and Russian ancestry. In essence, those selected for the survey were felt to be, 

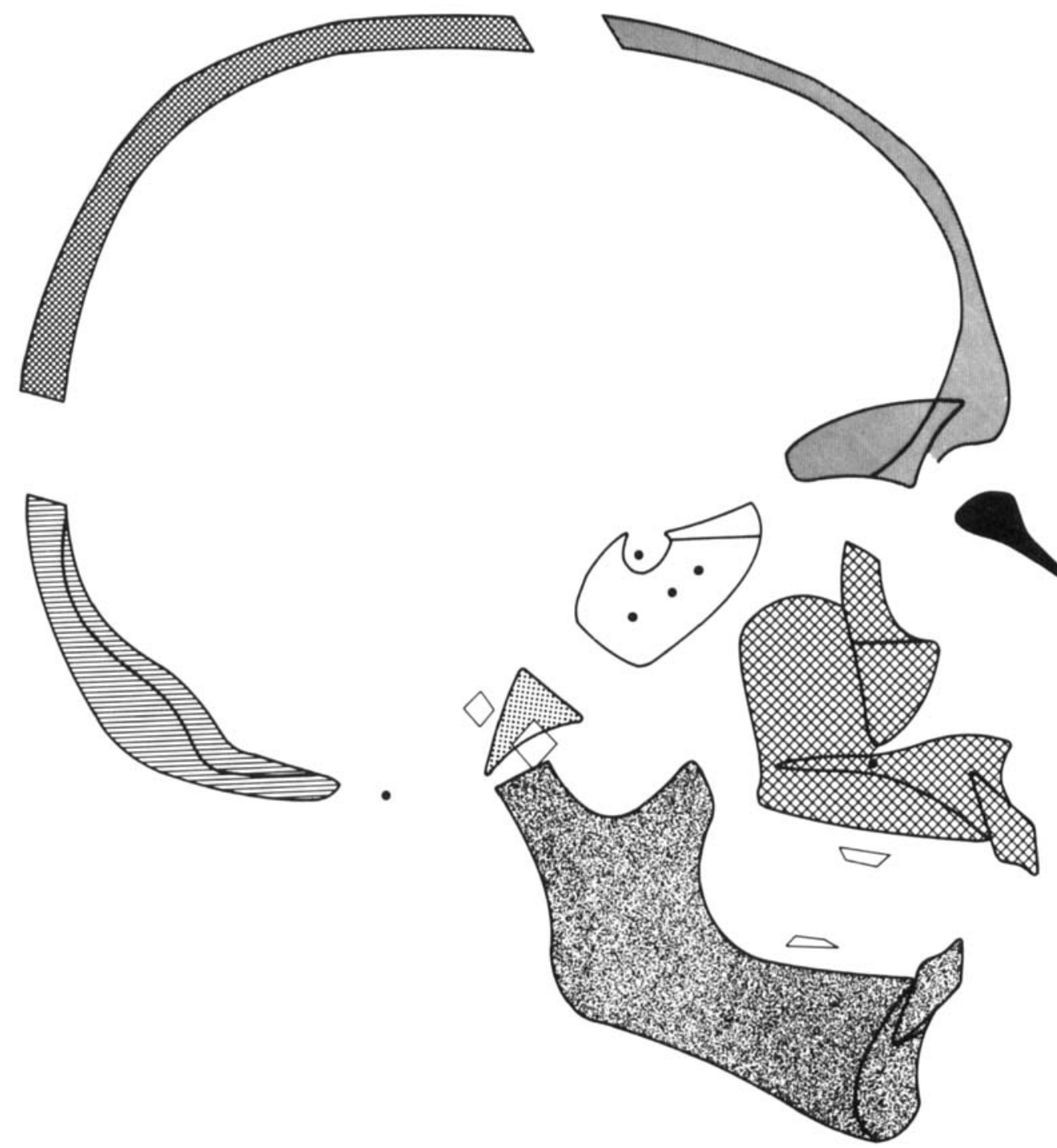

Fig. 1 The morphological structures comprising the mathematical model.

“. . . reasonably representative of the [white] children of the city of Philadelphia," during the period studied.

The cephalograms of this group of "normals" were selected by one of the authors (GFW), who also devised the computerized method used in this study (Walker, '67) and supervised the transformation of the radiographic information into digitized form for ready access by statistical computing programs. The data were processed using a console- oriented statistical computing program, called CONSTAT, developed by the Statistical Research Laboratory at the University of Michigan. The roentgenographic cephalograms of the children included in the study were reduced to this mathematical model by the following procedure:

(1) The outer contours of the individual skull bones were traced by experienced technicians to produce the sagittal profile of the craniofacial complex. Figure 1 illustrates the actual structures 
traced and hence the components of craniofacial morphology which may be analyzed using our model.

(2) The conventional (Krogman and Sassouni, '57) anthropometric points were marked, plus additional intermediate points to provide a sufficient number of coordinates to describe the skull contours with reasonable fidelity. These additional points were derived by a simple geometric division of the contours into two, four or eight segments depending on the distance between the anatomical points in question. For example, the contour between lambda and bregma is relatively long and so is divided into eight segments; from menton to gonion, four segments are sufficient. In all the model is comprised of 177 points and each point is equivalent, i.e., in the same anatomical position, for each skull. Figure 2 illustrates the positions of the 177 points on the tracing of a typical cephalogram. Nasion is point 58, A-point is 133 and B-point is 137 .

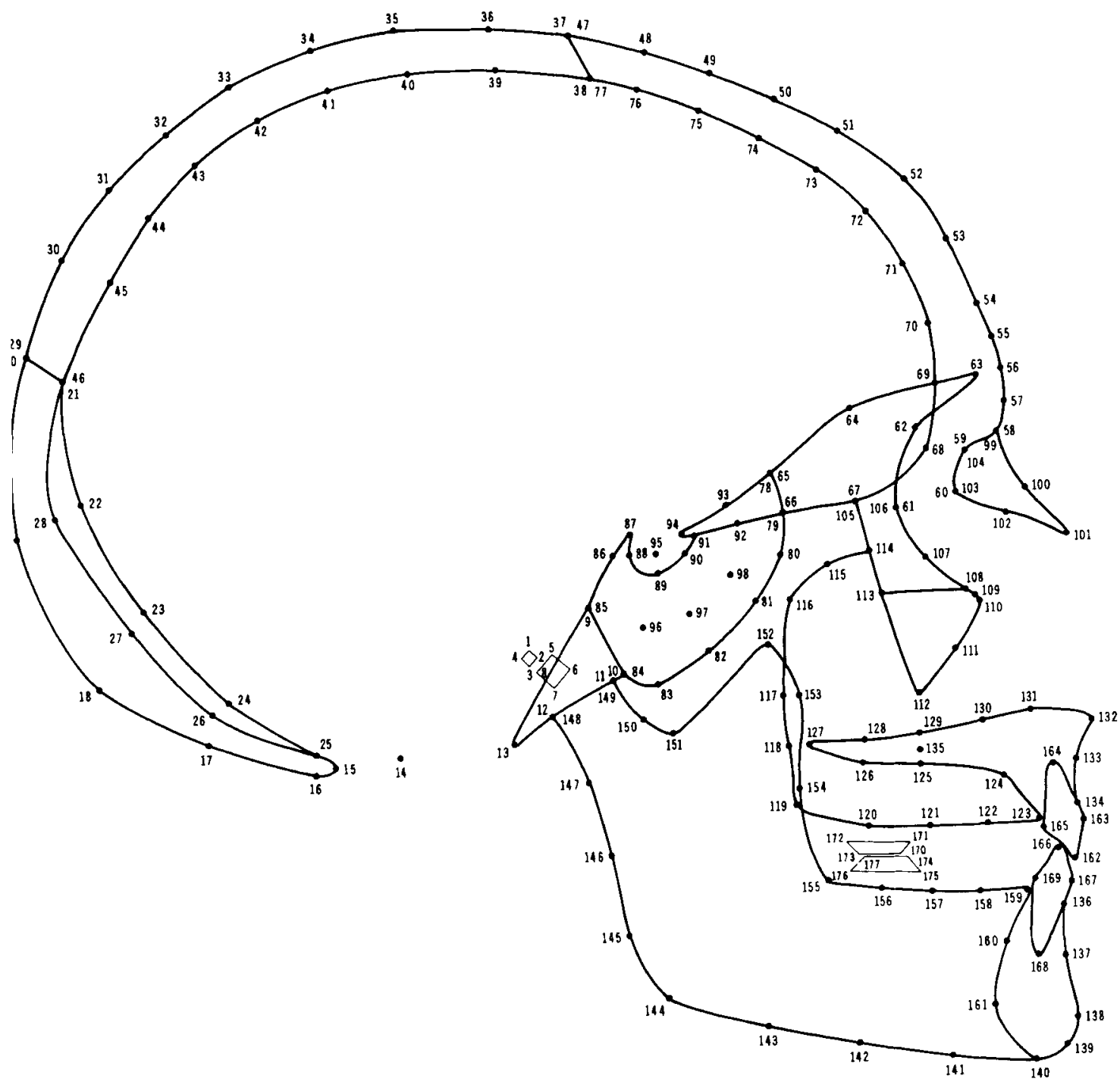

Fig. 2 The 177 coordinate points used to describe craniofacial morphology. 
(3) When the tracing is thus marked or "digitized" it is converted to numerical coordinates by a semi-automatic scanning process using an electronic device, such as the Benson-Lehner "OSCAR" (Walker, '67), and these coordinate values are automatically punched on IBM cards. We have found that a deck of 25 cards is sufficient to hold the 177 coordinate points and appropriate demographic information. This information may then be stored as cards or converted to magnetic tape and thus be available for processing on a digital computer.

An extremely rich data base can now be generated from these coordinate values: Lines, planes, projections, areas and angles are immediately available by the elementary use of coordinate geometry. Statistical analyses can then be performed on various sets of measurements of interest. While in this paper we concentrate on but a few of the available measurements, it should be noted that the model is much more general and can be used in a variety of studies of this kind; indeed, most of the standard orthodontic cephalometric analyses are immediately available as particular subsets of the measurements which can be generated from this model. Of course, it must be realized that the reliability of such analyses is dependent upon the reliability of the measurements themselves. In recognition of this, considerable care was taken with the measuring procedure employed in order to minimize the magnitudes of the measurement errors and, when possible, to obtain accurate estimates of these errors. The results of these studies are to be reported elsewhere; we remark here only that the "acid test" of whether or not the procedure is workable in practice is to check if the recorded coordinates of the mathematical model accurately reflect the information contained in the tracing and $x$-ray film. This can be done by simply having the computer plot the values for comparison (Walker, '67). These plots are scaled to be comparable to the tracing and $x$-ray and these may be superimposed to check on the "goodness of fit." We considered the recorded coordinates as acceptable if the plot was within about $0.5 \mathrm{~mm}$ of the tracing and/ or $\mathrm{x}$-ray. Thus the $\mathrm{N}=802$ subjects in the study were selected on the basis of having (a) "normal" occlusion and (b) accurate numerical representation in the mathematical model.

\section{RESULTS}

The $\mathrm{N}=802$ acceptable data files were stored in the memory of the computer (an IBM model 360/67) and the ANB angle for each subject computed. Table 1 gives the sample size, mean, variance, standard deviation, minimum and maximum values of the ANB angle (measured in degrees) for the males in the sample for several age groups. The age intervals are closed on the left and open on the right, e.g., the interval six to ten includes all those individuals in the sample who have attained their sixth birthday but have not yet celebrated their tenth. Table 2 gives the corresponding information for the females in the sample.

A glance at these tables is enough to show that, in the population studied, the "typical" value of the ANB angle is quite different from $2^{\circ}$; the overall mean being more in the neighborhood of $4.5^{\circ}$. (The 380 males had a mean of $4.67^{\circ}$; the 422 females a mean of $4.36^{\circ}$ ). This is not to say that an angle of $2^{\circ}$ is not in some sense "better," but indicates that "normal" (by Krogman's standards) individuals do not, on the average, attain this ideal. One may continue to insist that $2^{\circ}$ is the ideal value for the ANB angle, but should realized that the great majority of "normal" individuals simply do not look this way. This result has obvious implications for the setting-up of treatment goals; it may simply be asking too much to attain the "ideal" of $2^{\circ}$. Orthodontists may have to think more in terms of "acceptable compromises" (Steiner, '59) and to study additional variables recognizing that "normality" is a multivariate phenomenon, depending on proper combinations of measurements, as suggested by Wylie ('44).

We turn now to the questions of the dependence of the ANB angle on age and of sexual dimorphism. To test the hypothesis that the mean ANB angles for males are the same in each of the six age groups the Analysis of Variance was used. These means were significantly different $(\mathrm{P}<$ 
TABLE 1

Descriptive statistics for the distribution of the ANB angle in "Normal" males from 6 to 26 years of age

\begin{tabular}{rrrcccc}
\hline \multicolumn{1}{c}{ Age } & N & Mean & Variance & Std. Dev. & Minimum & Maximum \\
\hline $6-10$ & 42 & 5.170 & 4.311 & 2.076 & 1.220 & 9.740 \\
$10-12$ & 91 & 5.087 & 3.612 & 1.901 & 0.310 & 9.340 \\
$12-14$ & 113 & 4.929 & 3.746 & 1.935 & 0.500 & 9.770 \\
$14-16$ & 78 & 4.221 & 3.514 & 1.875 & 0.070 & 8.180 \\
$16-18$ & 34 & 3.879 & 2.149 & 1.466 & 0.340 & 5.900 \\
$18-26$ & 22 & 3.440 & 3.669 & 1.915 & 0.140 & 6.670 \\
Total & 380 & 4.668 & 3.811 & 1.952 & 0.070 & 9.770 \\
sample & 380 & & & & \\
\hline
\end{tabular}

TABLE 2

Descriptive statistics for the distribution of the ANB angle in "Normal" females from 6 to 26 years of age

\begin{tabular}{rrrrrrr}
\hline \multicolumn{1}{c}{ Age } & \multicolumn{1}{c}{$\mathrm{N}$} & Mean & Variance & Std. Dev. & Minimum & Maximum \\
\hline $6-10$ & 42 & 4.230 & 5.857 & 2.420 & 0.100 & 10.490 \\
$10-12$ & 105 & 4.606 & 5.096 & 2.257 & 0.330 & 10.640 \\
$12-14$ & 119 & 4.210 & 4.922 & 2.219 & 0.060 & 9.660 \\
$14-16$ & 92 & 4.242 & 4.695 & 2.167 & 0.660 & 10.880 \\
$16-18$ & 54 & 4.456 & 3.218 & 1.794 & 0.250 & 8.660 \\
$18-26$ & 10 & 4.763 & 5.908 & 2.431 & 0.640 & 8.530 \\
Total & 422 & 4.362 & 4.786 & 2.188 & 0.060 & 10.880 \\
sample & 422 & &
\end{tabular}

0.001 ) and subsequent investigation revealed a definite tendency for the ANB angle to decrease with increasing age. Since Bartlett's test accepted the hypotheesis of homogeneity of variance $(P>0.3)$ and since histograms of the ANB angle supported the assumption of normality (a typical example is given in fig. 3) we can only conclude that, for the male sample, the ANB angle has a definite tendency to decrease with increasing age.

The corresponding analysis of the female data exhibited no such differences; one cannot reject the hypothesis ( $\mathrm{P}>$ 0.25 ) that the mean ANB angles for females are the same for each of the age groups considered. Thus there appears to be a considerable amount of sexual dimorphism associated with the ANB angle in the population studied. The males have slightly larger ANB angles up to about 15 years of age when a reversal occurs, the males then tending to have smaller ANB angles than the females. While the ANB angle for females remains relatively constant from 6 to 26 years of age, there is a definite tendency for males to exhibit a decreasing ANB angle with increasing age.
This finding naturally raises the question of the structural dynamics of this difference in growth pattern. Using computer programs (Walker and Kowalski, '71) which allow us to extract any measurement (lines, angles, areas, etc.) definable in the context of the 177 points comprising our mathematical model, and after studying the growth curves of a number of these variables, we were able

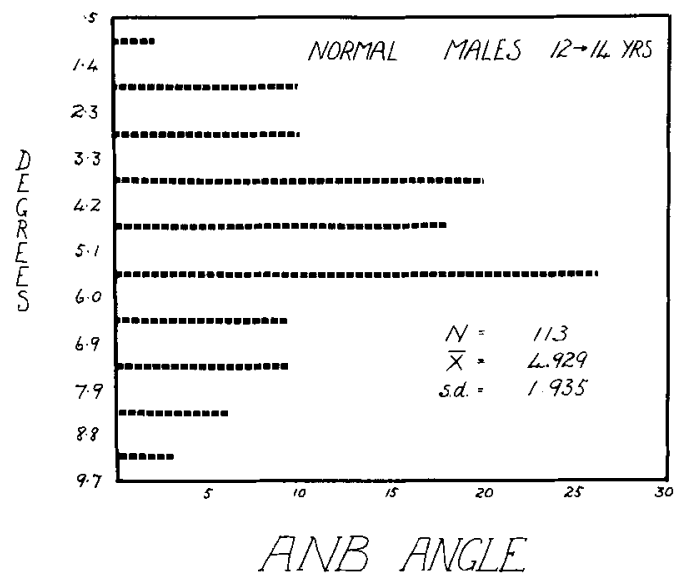

Fig. 3 Histogram of the ANB angle for males 12-14 years of age. 
to explain the observed difference in terms of mandibular growth: In the male, the mandible continues to grow steadily after puberty whereas this tendency is not exhibited in the female, as is illustrated in figure 4 where the growth curves for two definitions of mandibular length are presented. Irrespective of which definition of mandibular length is used it can be seen that up to about 12 years of age mandibular morphology and growth in the two sexes exhibit remarkable parallelism, but lower facial growth continues in the male well into the late teens. This prolonged growth of the mandible (relative to the maxillary structures) causes the closure of the ANB angle to occur after puberty and has direct relevance to decisions regarding orthodontic diagnosis and treatment as well as to the computation of morphological standards for the population studied.

\section{CONCLUSIONS}

We have seen that the mean of the ANB angle for the 380 males in the study is $4.67^{\circ}$ and for the 422 females it is $4.36^{\circ}$. For the combined sample, then, we have a mean ANB angle of the order of $4.5^{\circ}$ based on over 800 cases. This is considerably different from the "standard" of $2^{\circ}$ proposed by Steiner (59) and serves to illustrate the fact that orthodontic "ideals" are seldom realized in natural populations and should not be used uncritically in the study of racial variations.

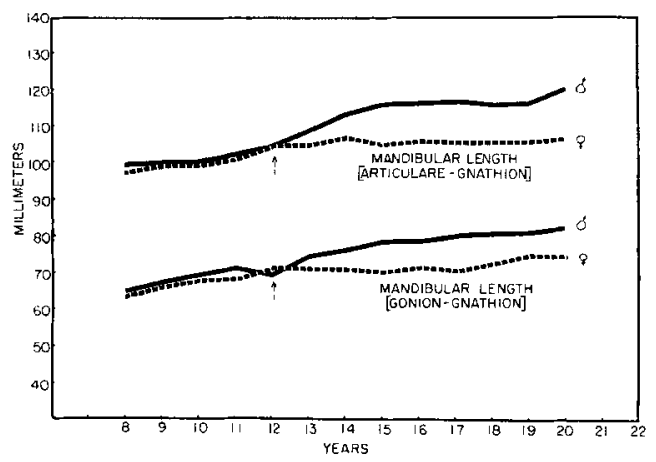

Fig. 4 Sex-specific growth curves for two measures of mandibular length. The arrows indicate the age at which the growth curves begin to exhibit sex differences.
We have also been able to identify a definite pattern of sexual dimorphism and to pinpoint the factors involved in the structural dynamics of the observed differences. These findings reinforce Steiner's (59) statement to the effect that age, sex and growth potential should be taken into account when studying measures of prognathism and provide some definitive information regarding just how these considerations should be implemented in practice. It might also be noted that despite the fact that angular measurements are often employed in cephalometric studies on the strength of the assumption that they are usually age-independent, this assumption is not tenable in the situation considered in this paper.

The results of this paper were obtained using a mathematical model of craniofacial morphology which may be used in a variety of contexts different from the particular study reported here. We conclude this discussion by suggesting that this model has the following advantages:

(1) The coordinate structure contains a considerable amount of information regarding both size and shape. Indeed, given this structure, our plotting programs have allowed us essentially to reproduce the morphological structures illustrated in figure 1. Thus the model has the advantage that, while retaining the descriptive power of the cephalogram, a numerical structure is introduced so that mathematical manipulations and statistical analyses may be applied.

(2) The model allows both numerical and visual checks to facilitate quality control of the data. We have programs that automatically "flag" extreme observations and produce plots of the suspected data file so that gross errors are easily identified and corrected.

(3) The data may be stored as punched cards or magnetic tape and so are available for rapid access by a computer. This allows on-line editing of the data bank and sequential analyses in the sense that should early results indicate the need to study more (or fewer) variables, these may be extracted and incorporated into the analysis.

(4) Statistical analyses are easily performed either on the coordinate values themselves or on derived measurements 
such as lengths, areas, planes, projections, angles, etc. Once the coordinate values are stored in a data file, the appropriate measurements may be extracted and the statistical analyses done in a matter of minutes.

(5) Many of the results may be presented graphically. An important benefit is the ability of the anthropologist, clinician and statistician alike to view the results and interpret the observed variations as a unified whole (all the structures depicted in fig. 1 are available) in a medium which is readily understood by all.

\section{LITERATURE CITED}

Krogman, W. M., and V. Sassouni 1957 A Syl labus in Roentgenographic Cephalometry. Phila delphia Center for Research in Child Growth, Philadelphia.

Steiner, C. C. 1959 Cephalometrics in clinical practice. Angle Orthodont., 29: 8-29.

Walker, G. F. 1967 Summary of a research report on the analysis of craniofacial growth. New Zealand Dent. J., 63: 31-38.

Walker, G. F., and C. J. Kowalski 1971 A Twodimensional coordinate model for the quantification, description, analysis, prediction and simulation of craniofacial growth. Growth, 35: 191-211.

Wylie, W. L. 1944 A quantitative method for the comparison of craniofacial patterns in different individuals; its application to a study of parents and offspring. Am. J. Anat., 74: 39-60. 